\title{
Measurement methods of total organic carbon in seawater
}

\author{
Shuwei Zhang ${ }^{1}$, Zhaoyu Wang ${ }^{1}$, Xuejiao Yan ${ }^{2}$, Jing Wang ${ }^{3}$, Li Zhang ${ }^{1}$, Qian Shi ${ }^{1}$, Da Yuan ${ }^{1}$, Ran Ma ${ }^{1}$ and Yan Liu ${ }^{1, a}$ \\ ${ }^{1}$ Institute of oceanographic Instrumentation, Qilu University of Technology (Shandong Academy of Sciences), Shandong Provincial Key \\ Laboratory of Ocean Environmental Monitoring Technology, National Engineering and Technological Research Center of Marine \\ Monitoring Equipment, Qingdao 266061, China \\ ${ }^{2}$ Qingdao Fisheries Technology Extension Station, Qingdao 266071, China \\ ${ }^{3}$ Third Institute of Oceanography, Ministry of Natural Resources, Xiamen 361005, China
}

\begin{abstract}
Total organic carbon (TOC) can reflect the total amount of organic matter in water. This paper introduces the common methods of measuring organic carbon, including high temperature combustion method, potassium persulfate oxidation method, spectrometry, ozone oxidation chemiluminescence method, supercritical water oxidation method and so on. At present, high temperature combustion method is the most widely used method for TOC measurement in seawater, because of its high oxidation efficiency. TOC sensor needs to be developed to realize in-situ and long-term monitoring.
\end{abstract}

\section{Introduction}

Total organic carbon (TOC) is an indicator of the total amount of organic matter in water. It is an important monitoring parameter of marine ecological environment, and can more reasonably indicate the degree of water pollution by organic matter [1-2]. The in-situ, rapid and long-term monitoring of TOC in seawater can timely understand the degree of marine organic pollution, and it is convenient to take immediate measures to control the development of marine pollution. It is the basis for early warning, prevention and control of marine pollution. At the same time, TOC, as a kind of biogenic element, can reflect the situation of life activities in water, and also plays an important role in the study of global carbon cycle [3-4].

At present, the monitoring of marine ecological environment in the world has formed a comprehensive system using ships, buoys, stations and other platforms. On site rapid monitoring instruments have become an important means to obtain data. The research and application of seawater TOC on-line monitoring technology has been paid great attention. It is imperative to strengthen the monitoring ability of TOC in seawater. This paper summarizes the representative measurement methods of TOC in seawater.

\section{Potassium persulfate oxidation method}

The measurement of organic carbon in seawater began with Natterer, who measured the seawater near Greece 100 years ago [5]. In the early stage, $\mathrm{K}_{2} \mathrm{Cr}_{2} \mathrm{O}_{7}, \mathrm{Ag}_{2} \mathrm{Cr}_{2} \mathrm{O}_{7}$, etc were used to oxidize organic carbon in water. These wet chemical oxidation processes are complex and timeconsuming. In 1964, Menzel et al. used persulfate as oxidant to oxidize organic matter, and the carbon dioxide produced was determined by non-dispersive infrared analyzer. This method is relatively simple and fast [6-7]. Later, Ehrbandt, Collins and other scholars proposed UV / potassium persulfate oxidation method [8]. The principle is that in the presence of potassium persulfate, the water sample is irradiated with high intensity ultraviolet light to decompose the organic matter and the $\mathrm{CO}_{2}$ produced was analyzed. UV / potassium persulfate oxidation and non-dispersive infrared detection technology realizes automatic and on-line analysis of total organic carbon. Representative instruments are produced by analytikjena company of Germany, Shimadzu Corporation of Japan and SERES Environnement company of France (Figure 1). However, the oxidation efficiency of this method is not ideal for complex organic compounds [9].

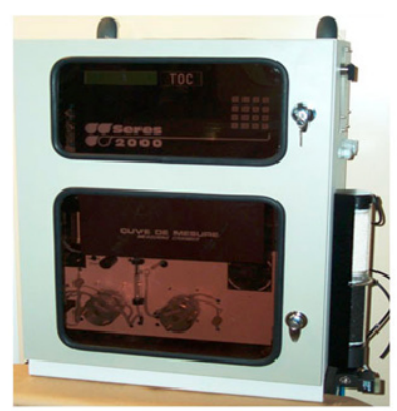

Figure 1. TOC on line automatic monitor produced by SERES Environnement company of France

\section{High temperature combustion method}

In 1985, Suzuki et al. proposed the high temperature combustion method, which was used to seawater in 1988

\footnotetext{
a Corresponding author: sdqdliuyan@126.com
} 
[10]. The core component of this method is combustion tube, which is filled with catalyst. After removing inorganic carbon, the water sample enters the combustion tube and evaporates instantly at high temperature. The organic matter in the water sample is burned and decomposed into carbon dioxide and water, and the salt in the water sample remains on the adsorbent in the tube. Driven by carrier gas, carbon dioxide is dried by condensing tube to remove water vapor, and then enters into non-dispersive infrared detector for quantitative analysis [11-13]. Because the high temperature combustion is relatively complete, the oxidation efficiency is high. And the measurement is fast, can realize automation. At present, the instrument based on high temperature combustion method has become the most used instrument for seawater TOC measurement. The instruments commonly used are the analyzers made by Shimadzu Corporation of Japan and Elementar company of Germany (Figure 2). However, carrier gas is required for measurement. Catalysts, usually platinum and other metal oxides, are expensive. Moreover, the catalyst is easy to be poisoned and blocked when measuring high salinity seawater, so it needs to be replaced frequently [14-15]. Therefore, this kind of instrument is mainly used for the measurement of seawater samples in the laboratory, and is not suitable for marine field use.

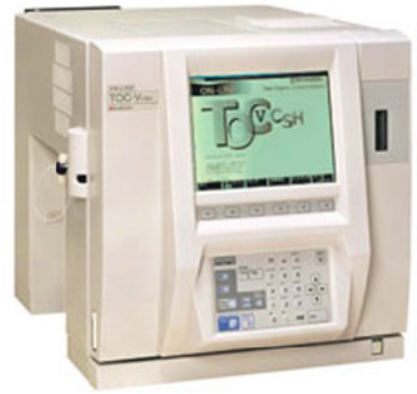

Figure 2. TOC analyzer produced by Shimadzu Corporation of Japan

\section{Spectrometry}

The organic carbon measurement instrument based on spectrometry calculates the content of organic carbon by measuring the absorption spectrum and fluorescence spectrum of organic matter in water [16]. This kind of instrument needs no chemical reagent, measures rapidly, and can realize in-situ and long-term monitoring. Representative instruments are sensors of Austrian s::can company and German TriOS company (Figure 3). It is worth noting that this kind of instrument measures the content of dissolved organic matter, which has ultraviolet absorption. There is no response to the organic matter which is not absorbed in the near ultraviolet region. In addition, the number of groups with UV absorption was measured by spectroscopic method. TOC is the total amount of carbon in organic matter. Therefore, there is a certain gap between the measured results of spectrometry and the actual content of total organic carbon in water [17]. Spectral organic carbon sensor should to be calibrated before use. It is suitable for qualitative monitoring the change trend of organic matter in water.

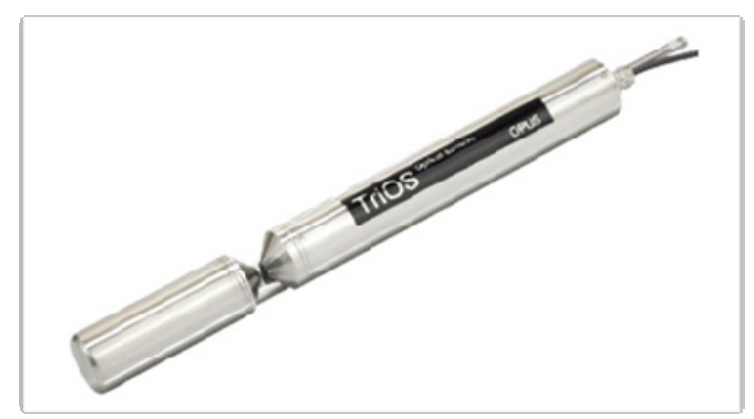

Figure 3. TriOS OPUS spectrum analyzer

\section{Other TOC measurement methods}

Ozone oxidation chemiluminescence method uses ozone as a strong oxidant to react with organic matter in seawater. The chemiluminescence signal generated in the reaction process is collected by photomultiplier tube, and then the TOC is calculated [18-19]. The instrument based on this method (Figure 4) does not need additional reagents, can avoid secondary pollution, and has a fast response speed. It is suitable for long-term use on stations, survey ships and other platforms. The results of ozone oxidation chemiluminescence method were close to those of high temperature combustion method. The luminescence mechanism of the reaction between ozone and organic compounds needs further study.

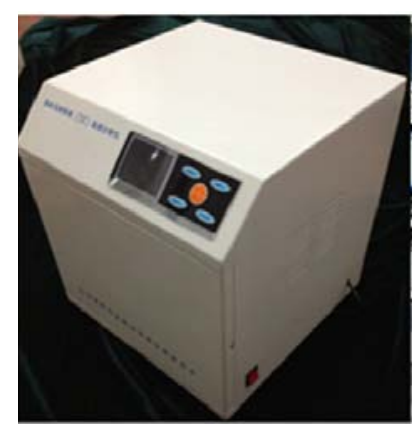

Figure 4. TOC on line automatic monitor based by ozone oxidation chemiluminescence method

Instruments made by UV oxidation and conductivity detection technology, such as products of GE Analysis Instruments and Mettler-Toledo company (Figure 5), can measure the total organic carbon on line, mainly used for the analysis of pure water and ultra-pure water samples. The instruments based on supercritical water oxidation technology need to provide high temperature and high pressure environment (Figure 5). They are not suitable for on-line measurement of seawater samples. They are mostly used for the determination of fresh water samples such as biological, pharmaceutical and industrial wastewater. 

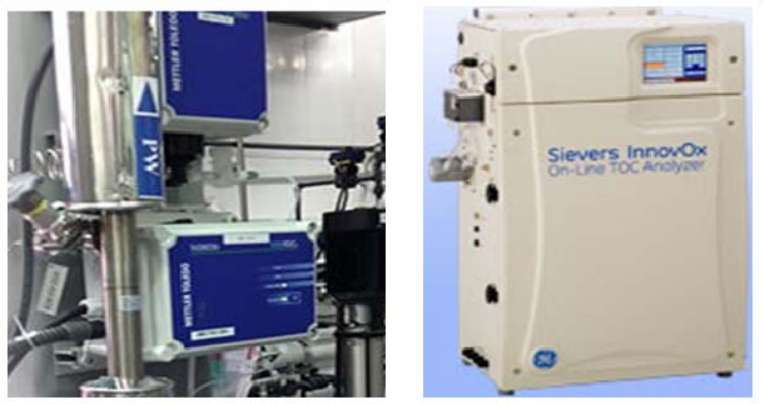

Figure 5. TOC automatic measuring instrument based by UV oxidation and conductivity detection technology and supercritical water oxidation technology

\section{Conclusion}

The commonly used measurement methods of TOC in water body are summarized in Table 1. At present, the measurement of TOC in seawater mainly adopts high temperature combustion method [20-21]. The samples are collected and brought back to the laboratory for analysis. Due to the problems of low oxidation efficiency, complex system, catalyst poisoning, frequent maintenance and high energy consumption, the existing methods are not suitable for in-situ and long-term monitoring of seawater TOC.

Table 1. Commonly used TOC measurement methods in water

Method Evaluation

High temperature combustion method persulfate oxidation method

Spectrometry
The oxidation efficiency of this method is high . The catalyst is easy to be poisoned and blocked when measuring high salinity seawater, and needs to be replaced frequently. This kind of instrument is mainly used for the measurement of seawater samples in the laboratory.

This kind of instrument can automatically and online measure TOC in water. The oxidation efficiency of this method is not ideal for complex organic compounds.

This kind of sensor measures rapidly, and can realize in-situ and long-term monitoring. It is suitable for qualitative monitoring the change trend of organic matter in water.
Ozone oxidation chemiluminescence method oxidation method

UV oxidation and conductivity detection method

In the future, TOC on-line monitoring instrument will be miniaturized, intelligent and low-power consumption. The future trend is to develop seawater TOC sensor, which can realize in-situ, real-time and long-term monitoring. However, the low concentration of TOC in seawater, complex matrix, large inorganic interference and oxidation efficiency should be considered.

\section{Acknowledgments}

This work was supported by Key Research and Development Program of Shandong Province (No.2019GHY112070, No.2019GHY112054), the Joint Funds of the National Natural Science Foundation of China (Grant No.U1806202), Natural Science Foundation of Shandong Province (No.ZR2018ZB0523, No. ZR2019BD043) and Qingdao applied basic research program (No. 19-6-2-26-cg).

\section{References}

1. R. A. Houghton, Annu. Rev. Earth Planet. Sci. 35, 313- 347 (2007)

2. D. K. Steinberg, S. A. Goldthwait, D. A. Hansell, Deep-Sea Res. Pt. I 49, 1445-1461 (2002)

3. O. Hiroshi, U. Toshihiro, K. Isao, Deep-Sea Res. Pt. II 50, 353-366 (2003)

4. D. A. Hansell, C. A. Carlson, and R. Schlitzer, Global Biogeochem. Cy. 26, GB1016 (2012)

5. J. Wang, L. Tan. Mar. Sci. 2, 26 (1999)

6. J. H. McKenna, P. H. Doering, Mar. Chem. 48, 109114 (1995)

7. J. H. Sharp, Mar. Chem. 11, 211-229 (1973)

8. J. T. Wang, Z. B. Zhang, L. S. Liu, Chin. J. Oceanol. Limnol. 15, 25-31 (1997)

9. J. J. Ridal, R. M. Moore, Mar. Chem. 3-4, 167-188 (1993) 
10. Y. Suzuki, E. Tanoue and I. Hiroshi, Deep-Sea Res. 39, 185-198 (1992)

11. J. H. Sharp, R. Benner, L. Bennett, C. A. Carlson, S. E. Fitzwater, E. T. Peltzer, L. M. Tupas, Mar. Chem. 48, 91-108 (1995)

12. W. Chen, Z. Zhao, J. F. Koprivnjak, E. M. Perdue, Mar. Chem. 78, 185- 196 (2002)

13. J. Qian, K .Mopper, Anal. Chem., 68, 3090- 3097 (1996)

14. G. Cauwet, Mar. Chem., 47, 55-64 (1994)

15. M. L. Peterson, S. Q. Lang, A. K. Aufdenkampe, J. I. Hedges, Mar. Chem. 81, 89-104 (2003)

16. B. Coulomb, Y. Richardson, C. Brach-Papa, J. L. Boudenne, F. Theraulaz, Iin. J. Environ. An. Ch. 86, 1079-1093 (2006)

17. Y. Yan, S. Du, Y. Wu, Chin. J. Environ. Eng. 5, 521-522 (2011)

18. G. Ren, Y. Tang, R. Ma, Y. Liu, Adv. Mat. Res. 482-484, 224-228 (2012)

19. Y. Liu, P. Fan, G. Hou, J. Sun, Y. Cheng, R. Ma, Adv. Mat. Res. 468-471, 2842-2848 (2012)

20. L. M. Tupas, B. N. Popp, M. K. David, Mar. Chem. 3, 207-216 (1994)

21. J. H. Sharp, C. A. Carlson, E. T. Peltzer, D. M. Castle-Ward, K. R. Rinker, Mar. Chem. 77, 239-253 (2002) 